CARPATHIAN J. MATH.

Volume 37 (2021), No. 3,

Pages 463 - 476
Online version at https : //www . carpathian. cunbm. utcluj. ro/

Print Edition: ISSN 1584 - 2851; Online Edition: ISSN 1843 - 4401

DOI: https://doi.org/10.37193/CJM.2021.03.09

\title{
Resolvents of equilibrium problems on a complete geodesic space with curvature bounded above
}

\author{
YASUNORI KIMURA
}

ABSTRACT. We consider equilibrium problems on a complete geodesic space with curvature bounded above by one and propose the notion of resolvents for this problem. We prove its well-definedness as a single-valued mapping whose domain is the whole space, and its geometric properties.

\section{INTRODUCTION}

Let $K$ be a nonempty set and $f: K \times K \rightarrow \mathbb{R}$ be a bifunction on $K$. An equilibrium problem for $f$ is defined as to find $z_{0} \in K$ such that

$$
f\left(z_{0}, y\right) \geq 0
$$

for every $y \in K$. Equilibrium problems were first studied intensively by Blum and Oettli [3] in the setting of topological vector spaces and Banach spaces. They also proposed the notion of resolvents of a bifunction for an equilibrium problem and showed that its domain is the whole space.

Further study of this problem and properties of resolvents in the setting of Hilbert spaces have been done by Combettes and Hirstoaga [5]; they considered a countable system of equilibrium problems and obtained several important properties of the resolvents. Due to some nice properties of resolvents, we may apply many kinds of results in fixed point theory to this problem such as existence theorems and approximation theorems of its solutions.

In 2018, Kimura and Kishi [9] proposed the notion of resolvents of a bifunction defined on a Hadamard space. They assumed the convex hull finite property for the underlying space and, by using a similar technique to that in [3], they obtained several properties of resolvents. For the study of equilibrium problems on Hadamard spaces, another approach can be found in [13].

In this paper, we consider an equilibrium problem defined on an admissible complete CAT(1) space. The class of CAT(1) spaces includes that of Hadamard spaces, however, geometric properties of CAT(1) spaces are totally different from those of Hadamard spaces. We propose the notion of resolvents of a bifunction for an equilibrium problem by using the logarithmic cosine function. We show its well-definedness as a single-valued mapping defined on the whole space, and its spherical nonspreadingness of sum type, under the assumption called the convex hull finite property. We also show several related results obtained from fixed point theory on geodesic spaces.

\section{PRELIMINARIES}

A CAT(1) space is defined as a uniquely $\pi$-geodesic metric space satisfying the CAT(1) inequality with the two-dimensional unit sphere $\mathbb{S}^{2}$ as a model space. Namely, any two

Received: 01.10.2020. In revised form: 23.03.2021. Accepted: 01.04.2021

2010 Mathematics Subject Classification. 90C33, 90C48, 49K27.

Key words and phrases. equilibruim problem, resolvent, $C A T(1)$ space. 
points $x, y$ in a CAT(1) space $X$ with $d(x, y)<\pi$ can be connected with a unique geodesic $c:[0, d(x, y)] \rightarrow X$, and for any $x, y, z \in X$ with $d(x, y)+d(y, z)+d(z, x)<2 \pi, p, q \in$ $\triangle(x, y, z) \subset X$ and their comparison points $\bar{p}, \bar{q} \subset \triangle(\bar{x}, \bar{y}, \bar{z}) \subset \mathbb{S}^{2}$ satisfy

$$
d(p, q) \leq d_{\mathbb{S}^{2}}(\bar{p}, \bar{q}) .
$$

Since the geodesic $c$ between $x, y$ is uniquely defined if $d(x, y)<\pi$, for any $z \in[x, y]=$ $c([0, d(x, y)])$, we can find $t \in[0,1]$ such that $d(x, z)=(1-t) d(x, y)$ and $d(z, y)=t d(x, y)$. We denote it by $z=t x \oplus(1-t) y$ and we call it a convex combination of $x$ and $y$. In what follows, we usually suppose that $X$ is an admissible CAT(1) space, that is, $d(x, y)<\pi / 2$ for every $x, y \in X$. In an admissible CAT(1) space, every two points have a unique geodesic [4, Proposition II.1.4].

For a subset $A$ of a metric space $X$, we denote its closure by $\operatorname{cl} A$ and its diameter by $\operatorname{diam} A$. Suppose that $X$ is an admissible CAT(1) space. The convex hull $\operatorname{co} A$ of $A$ is defined by

$$
\operatorname{co} A=\bigcup_{j=1}^{\infty} F_{j},
$$

where $F_{1}=A$ and $F_{j+1}=\left\{t u_{j} \oplus(1-t) u_{j}^{\prime} \in X \mid u_{j}, u_{j}^{\prime} \in F_{j}, t \in[0,1]\right\}$ for $j \in \mathbb{N}$.

The following result is a fundamental property of CAT(1) spaces derived from the spherical law of cosines; see, for example, [4, 11].

Theorem 2.1. Let $X$ be a CAT(1) space and suppose $x, y, z \in X$ satisfy $d(x, y)+d(y, z)+$ $d(z, x)<2 \pi$. Then,

$$
\cos d(t x \oplus(1-t) y, z) \sin d(x, y) \geq \cos d(x, z) \sin (t d(x, y))+\cos d(y, z) \sin ((1-t) d(x, y))
$$

for $t \in[0,1]$.

From this theorem, we can easily deduce the following inequalities.

Theorem 2.2 (Kimura-Kohsaka [10], Kimura-Satô [11]). Let $X$ be a CAT(1) space and suppose $x, y, z \in X$ satisfy $d(x, y)+d(y, z)+d(z, x)<2 \pi$. Then,

$$
\cos d\left(\frac{1}{2} x \oplus \frac{1}{2} y, z\right) \cos \left(\frac{1}{2} d(x, y)\right) \geq \frac{1}{2} \cos d(x, z)+\frac{1}{2} \cos d(y, z),
$$

and

$$
\cos d(t x \oplus(1-t) y, z) \geq t \cos d(x, z)+(1-t) \cos d(y, z)
$$

for $t \in[0,1]$.

Let $K$ be a nonempty closed convex subset of an admissible CAT(1) space $X$. For $x \in X$, there exists a unique point $u_{x} \in K$ which is nearest to $x$, that is, $d\left(x, u_{x}\right)=$ $\inf _{z \in K} d(x, z)$. We define a mapping $P_{K}: X \rightarrow K$ by $P_{K} x=u_{x}$ for each $x \in X$ and call it a metric projection onto $K$. In this case, we know that

$$
\cos d\left(x, P_{K} x\right) \cos d\left(P_{K} x, z\right) \geq \cos d(x, z)
$$

for every $z \in K$.

For more details of CAT(1) spaces and their properties, see [4] for instance.

Let $X$ be an admissible CAT(1) space. A function $g: X \rightarrow \mathbb{R}$ is said to be convex if

$$
g(t x \oplus(1-t) y) \leq t g(x)+(1-t) g(y)
$$

for any $x, y \in X$ and $t \in] 0,1[$.

It is shown in [7] that a function $-\log \cos d(\cdot, z)$ is convex on an admissible CAT(1) space $X$. Indeed, by Theorem 2.2, we have

$$
\cos d(t x \oplus(1-t) y, z) \geq t \cos d(x, z)+(1-t) \cos d(y, z)
$$


and since $s \mapsto-\log s$ is decreasing and convex on $] 0,+\infty[$, we get

$-\log \cos d(t x \oplus(1-t) y, z) \leq-\log (t \cos d(x, y)+(1-t) \cos d(x, z))$

$$
\leq-t \log \cos d(x, y)-(1-t) \log \cos d(y, z)
$$

for $x, y \in X$ and $t \in] 0,1[$.

A function $g: X \rightarrow \mathbb{R}$ is said to be lower semicontinuous if

$$
g\left(x_{0}\right) \leq \liminf _{n \rightarrow \infty} g\left(x_{n}\right)
$$

for every $\left\{x_{n}\right\} \subset X$ converging to $x_{0} \in X$. We say that $g$ is upper hemicontinuous if

$$
g(y) \geq \limsup _{t \rightarrow 0} g(t x \oplus(1-t) y)
$$

for every $x, y \in X$.

Theorem 2.3 (Kimura-Kohsaka [10]). Every lower semicontinuous convex function of an admissible complete CAT(1) space into $\mathbb{R}$ is bounded below.

Let $X$ be an admissible complete CAT(1) space. A mapping $T: X \rightarrow X$ is said to be spherically nonspreading of product type [10] if

$$
\cos ^{2} d(T x, T y) \geq \cos d(T x, y) \cos d(T y, x)
$$

for every $x, y \in X$. Notice that, in [10], they call it simply a spherically nonspreading mapping. Afterword, Kajimura and Kimura [7] proposed another type of nonspreadingness as below and they distinguished them by putting product and sum types.

The other notion of spherical nonspreadingness they proposed is as follows: $T$ is said to be spherically nonspreading of sum type [7] if

$$
2 \cos d(T x, T y) \geq \cos d(T x, y)+\cos d(T y, x)
$$

for every $x, y \in X$. It is easy to see [7] that if $T$ is spherically nonspreading of sum type, then it is also that of product type; for $T$ satisfying (2.2), we have

$$
\cos d(T x, T y) \geq \frac{\cos d(T x, y)+\cos d(T y, x)}{2} \geq \sqrt{\cos d(T x, y) \cos d(T y, x)},
$$

which implies (2.1).

For a mapping $T: X \rightarrow X$, the set of all fixed points of $T$ is denoted by $\operatorname{Fix} T$, that is,

$$
\operatorname{Fix} T=\{z \in X \mid z=T z\} .
$$

$T$ is said to be quasinonexpansive if Fix $T \neq \emptyset$ and $d(T x, z) \leq d(x, z)$ for every $x \in X$ and $z \in \operatorname{Fix} T$. It is known that Fix $T$ is closed and convex if $T$ is quasinonexpansive. Since every spherically nonspreading mapping of product type with a fixed point is quasinonexpansive [10], so is that of sum type.

Let $X$ be an admissible CAT(1) space and $K$ a nonempty closed convex subset of $X$. We consider an equilibrium problem for a bifunction $f: K \times K \rightarrow \mathbb{R}$, that is, to find $z_{0} \in K$ such that

$$
f\left(z_{0}, y\right) \geq 0
$$

for every $y \in K$. The set of solutions to the equilibrium problem for $f$ is denoted by Equil $f$, that is

$$
\text { Equil } f=\left\{\begin{array}{l|l|}
z \in K & \inf _{y \in K} f(z, y) \geq 0
\end{array} .\right.
$$

Following [3,5], we suppose the four conditions for $f$ as follows.

(E1) $f(y, y)=0$ for all $y \in K$;

(E2) $f(y, z)+f(z, y) \leq 0$ for all $y, z \in K$;

(E3) $f(y, \cdot): K \rightarrow \mathbb{R}$ is lower semicontinuous and convex for every $y \in K$;

(E4) $f(\cdot, z): K \rightarrow \mathbb{R}$ is upper hemicontinuous for every $z \in K$. 


\section{THE KKM LEMMA ON CAT(1) SPACES}

The Knaster-Kuratowski-Mazurkiewicz lemma, or the KKM lemma in short, is one of the most important results in nonlinear analysis since it plays a crucial role in the proof of the Brouwer fixed point theorem. This lemma has been considered also in the setting of geodesic spaces; see $[1,8,14]$ and references therein. We focus on the result of Niculescu and Rovenţa [14], a generalized KKM lemma in the setting of complete CAT(0) spaces. To obtain it, they assumed the convex hull finite property for the underlying space.

Using a similar technique to their proof, in this section, we prove three lemmas corresponding to the KKM lemma in the setting of admissible complete CAT(1) spaces with the convex hull finite property.

Let $X$ be an admissible CAT(1) space. We say $X$ has the convex hull finite property [15] if every continuous selfmapping on cl co $E$ has a fixed point for every finite subset $E$ of $X$.

Lemma 3.1. Let $E=\left\{x_{1}, x_{2}, \ldots, x_{l}\right\}$ be a finite subset of an admissible complete CAT(1) space $X$ and $\left\{M_{1}, M_{2}, \ldots, M_{l}\right\}$ be a finite family of nonempty closed convex subsets of $X$ such that $\bigcap_{i=1}^{l} M_{i}=\emptyset$. For given $u \in \operatorname{clco} E$, define $g_{u}: \operatorname{clco} E \rightarrow[0,+\infty[$ by

$$
g_{u}(y)=-\frac{1}{\sigma(u)} \sum_{i=1}^{l} d\left(u, M_{i}\right) \log \cos d\left(y, x_{i}\right)
$$

for $y \in X$, where $\sigma(u)=\sum_{i=1}^{l} d\left(u, M_{i}\right)$. Then, $g_{u}$ has a unique minimizer $y_{u} \in$ clco $E$ for every $u \in X$, and the single-valued mapping $T: \operatorname{cl} \operatorname{co} E \rightarrow \operatorname{cl} \operatorname{co} E$ defined by $T u=y_{u}$ for every $u \in \mathrm{cl} \operatorname{co} E$ is continuous.

Proof. Fix $u \in X$ arbitrarily. Since $\bigcap_{i=1}^{l} M_{i}=\emptyset$, we have $\sigma(u)=\sum_{i=1}^{l} d\left(u, M_{i}\right)>0$. Let $\left\{y_{n}\right\}$ be a sequence in clco $E$ such that $\left\{g_{u}\left(y_{n}\right)\right\}$ is nonincreasing and converges to $L=\inf _{y \in \operatorname{cl} \operatorname{co} E} g_{u}(y)$. Let $m, n \in \mathbb{N}$ with $n \leq m$. By Theorem 2.2, we have

$$
\begin{aligned}
&-\log \cos d\left(\frac{1}{2} y_{m} \oplus \frac{1}{2} y_{n}, x_{i}\right)-\log \cos \left(\frac{1}{2} d\left(y_{m}, y_{n}\right)\right) \\
& \leq-\log \left(\frac{1}{2} \cos d\left(y_{m}, x_{i}\right)+\frac{1}{2} \cos d\left(y_{n}, x_{i}\right)\right)
\end{aligned}
$$

for $i=1,2, \ldots, l$. Since $t \mapsto-\log t$ is convex on $] 0,+\infty[$, we have

$$
\begin{aligned}
& L-\log \cos \left(\frac{1}{2} d\left(y_{m}, y_{n}\right)\right) \\
& \leq g_{u}\left(\frac{1}{2} y_{m} \oplus \frac{1}{2} y_{n}\right)-\log \cos \left(\frac{1}{2} d\left(y_{m}, y_{n}\right)\right) \\
& =-\frac{1}{\sigma(u)} \sum_{i=1}^{l} d\left(u, M_{i}\right)\left(\log \cos d\left(\frac{1}{2} y_{m} \oplus \frac{1}{2} y_{n}, x_{i}\right)+\log \cos \left(\frac{1}{2} d\left(y_{m}, y_{n}\right)\right)\right) \\
& \leq-\frac{1}{\sigma(u)} \sum_{i=1}^{l} d\left(u, M_{i}\right) \log \left(\frac{1}{2} \cos d\left(y_{m}, x_{i}\right)+\frac{1}{2} \cos d\left(y_{n}, x_{i}\right)\right) \\
& \leq-\frac{1}{2 \sigma(u)} \sum_{i=1}^{l} d\left(u, M_{i}\right) \log \cos d\left(y_{m}, x_{i}\right)-\frac{1}{2 \sigma(u)} \sum_{i=1}^{l} d\left(u, M_{i}\right) \log \cos d\left(y_{n}, x_{i}\right) \\
& =\frac{1}{2} g_{u}\left(y_{m}\right)+\frac{1}{2} g_{u}\left(y_{n}\right) .
\end{aligned}
$$


Since $g_{u}\left(y_{m}\right) \leq g_{u}\left(y_{n}\right)$, we obtain

$$
0 \leq-\log \cos \left(\frac{1}{2} d\left(y_{m}, y_{n}\right)\right) \leq \frac{1}{2} g_{u}\left(y_{m}\right)+\frac{1}{2} g_{u}\left(y_{n}\right)-L \leq g_{u}\left(y_{n}\right)-L \rightarrow 0
$$

as $n \rightarrow \infty$. This shows that $\left\{y_{n}\right\}$ is a Cauchy sequence and hence it has a limit $y_{0} \in \operatorname{cl} \operatorname{co} E$. Since $g_{u}$ is a continuous function, we have

$$
g_{u}\left(y_{0}\right)=g_{u}\left(\lim _{n \rightarrow \infty} y_{n}\right)=\lim _{n \rightarrow \infty} g_{u}\left(y_{n}\right)=L,
$$

which shows that $y_{0}$ is a minimizer of $g_{u}$. For the uniqueness of the minimizer of $g_{u}$, suppose that both $y_{0}$ and $y_{0}^{\prime}$ minimize $g_{u}$. Then, by the same calculation above, we have

$$
L-\log \cos \left(\frac{1}{2} d\left(y_{0}, y_{0}^{\prime}\right)\right) \leq \frac{1}{2} g_{u}\left(y_{0}\right)+\frac{1}{2} g_{u}\left(y_{0}^{\prime}\right) .
$$

It follows that

$$
0 \leq-\log \cos \left(\frac{1}{2} d\left(y_{0}, y_{0}^{\prime}\right)\right) \leq \frac{1}{2} g_{u}\left(y_{0}\right)+\frac{1}{2} g_{u}\left(y_{0}^{\prime}\right)-L=\frac{1}{2} L+\frac{1}{2} L-L=0
$$

and hence $-\log \cos \left(d\left(y_{0}, y_{0}^{\prime}\right) / 2\right)=0$, which is equivalent to $y_{0}=y_{0}^{\prime}$. Therefore the minimizer of $g_{u}$ is unique for every $u \in \operatorname{cl} \operatorname{co} E$.

To prove the continuity of $T$, we first show that the inequality

$$
\cos d(T u, T v) \geq \frac{1}{\sigma(u)} \sum_{i=1}^{l} d\left(u, M_{i}\right) \frac{\cos d\left(T u, x_{i}\right)}{\cos d\left(T v, x_{i}\right)}
$$

holds for any $u, v \in \operatorname{cl} \operatorname{co} E$. If $T u=T v$, then (3.3) obviously holds with equality. Thus we may assume $T u \neq T v$. From the definition of $T$, for $t \in] 0,1[$, we have

$$
\begin{aligned}
0 & \leq g_{u}((1-t) T u \oplus t T v)-g_{u}(T u) \\
& =\frac{1}{\sigma(u)} \sum_{i=1}^{l} d\left(u, M_{i}\right)\left(-\log \cos d\left((1-t) T u \oplus t T v, x_{i}\right)+\log \cos d\left(T u, x_{i}\right)\right) \\
& \leq \frac{1}{\sigma(u)} \sum_{i=1}^{l} d\left(u, M_{i}\right) L_{i}(t)
\end{aligned}
$$

where

$$
\begin{aligned}
L_{i}(t)= & -\log \left(\cos d\left(T u, x_{i}\right) \sin ((1-t) d(T u, T v))+\cos d\left(T v, x_{i}\right) \sin (t d(T u, T v))\right) \\
& +\log \sin d(T u, T v)+\log \cos d\left(T u, x_{i}\right),
\end{aligned}
$$

which is obtained by Theorem 2.1. Since $\lim _{t \downarrow 0} L_{i}(t)=0$ for $i=1,2, \ldots, l$, by l'Hospital's rule, we have

$$
\begin{aligned}
0 & \leq \lim _{t \downarrow 0} \frac{(1 / \sigma(u)) \sum_{i=1}^{l} d\left(u, M_{i}\right) L_{i}(t)}{t}=\lim _{t \downarrow 0} \frac{d}{d t}\left(\frac{1}{\sigma(u)} \sum_{i=1}^{l} d\left(u, M_{i}\right) L_{i}(t)\right) \\
& =\frac{1}{\sigma(u)} \sum_{i=1}^{l}\left(d\left(u, M_{i}\right) \lim _{t \downarrow 0} \frac{d L_{i}(t)}{d t}\right) .
\end{aligned}
$$

Further, we have

$$
\begin{aligned}
\frac{d L_{i}(t)}{d t}= & -d(T u, T v) \frac{-\cos d\left(T u, x_{i}\right) \cos ((1-t) d(T u, T v))+\cos d\left(T v, x_{i}\right) \cos (t d(T u, T v))}{\cos d\left(T u, x_{i}\right) \sin ((1-t) d(T u, T v))+\cos d\left(T v, x_{i}\right) \sin (t d(T u, T v))} \\
& \rightarrow \frac{d(T u, T v)\left(\cos d\left(T u, x_{i}\right) \cos d(T u, T v)-\cos d\left(T v, x_{i}\right)\right)}{\cos d\left(T u, x_{i}\right) \sin d(T u, T v)}
\end{aligned}
$$


as $t \downarrow 0$. Hence we have

$$
\begin{aligned}
0 & \leq \frac{1}{\sigma(u)} \sum_{i=1}^{l}\left(d\left(u, M_{i}\right) \lim _{t \rightarrow 0} \frac{d L_{i}(t)}{d t}\right) \\
& \leq \frac{1}{\sigma(u)} \sum_{i=1}^{l}\left(d\left(u, M_{i}\right) \frac{d(T u, T v)\left(\cos d\left(T u, x_{i}\right) \cos d(T u, T v)-\cos d\left(T v, x_{i}\right)\right)}{\cos d\left(T u, x_{i}\right) \sin d(T u, T v)}\right) \\
& =\frac{d(T u, T v)}{\sigma(u) \sin d(T u, T v)} \sum_{i=1}^{l} d\left(u, M_{i}\right)\left(\cos d(T u, T v)-\frac{\cos d\left(T v, x_{i}\right)}{\cos d\left(T u, x_{i}\right)}\right) \\
& =\frac{d(T u, T v)}{\sin d(T u, T v)}\left(\cos d(T u, T v)-\frac{1}{\sigma(u)} \sum_{i=1}^{l} d\left(u, M_{i}\right) \frac{\cos d\left(T v, x_{i}\right)}{\cos d\left(T u, x_{i}\right)}\right) .
\end{aligned}
$$

Since $d(T u, T v) / \sin d(T u, T v)>0$, we obtain the desired inequality (3.3).

Now we prove the continuity of $T$. Let $\left\{u_{n}\right\}$ be a sequence in clco $E$ and suppose that $u_{n} \rightarrow u_{0} \in \operatorname{clco} E$. Then, by (3.3) we have

$$
\cos d\left(T u_{n}, T u_{0}\right) \geq \frac{1}{\sigma\left(u_{n}\right)} \sum_{i=1}^{l} d\left(u_{n}, M_{i}\right) \frac{\cos d\left(T u_{n}, x_{i}\right)}{\cos d\left(T u_{0}, x_{i}\right)}=\sum_{i=1}^{l} \frac{d\left(u_{n}, M_{i}\right)}{\sigma\left(u_{n}\right)} \frac{\cos d\left(T u_{n}, x_{i}\right)}{\cos d\left(T u_{0}, x_{i}\right)} .
$$

We also have

$$
\begin{aligned}
\cos d\left(T u_{0}, T u_{n}\right) & \geq \frac{1}{\sigma\left(u_{0}\right)} \sum_{i=1}^{l} d\left(u_{0}, M_{i}\right) \frac{\cos d\left(T u_{0}, x_{i}\right)}{\cos d\left(T u_{n}, x_{i}\right)}=\sum_{i=1}^{l} \frac{d\left(u_{0}, M_{i}\right)}{\sigma\left(u_{0}\right)} \frac{\cos d\left(T u_{0}, x_{i}\right)}{\cos d\left(T u_{n}, x_{i}\right)} \\
& =\sum_{i=1}^{l}\left(\frac{d\left(u_{n}, M_{i}\right)}{\sigma\left(u_{n}\right)}-\Delta_{i}\left(u_{n}, u_{0}\right)\right) \frac{\cos d\left(T u_{0}, x_{i}\right)}{\cos d\left(T u_{n}, x_{i}\right)}
\end{aligned}
$$

where

$$
\Delta_{i}\left(u_{n}, u_{0}\right)=\frac{d\left(u_{n}, M_{i}\right)}{\sigma\left(u_{n}\right)}-\frac{d\left(u_{0}, M_{i}\right)}{\sigma\left(u_{0}\right)} .
$$

From these inequalities, we have

$$
\begin{aligned}
1 & \geq \cos d\left(T u_{n}, T u_{0}\right) \\
& \geq \frac{1}{2} \sum_{i=1}^{l} \frac{d\left(u_{n}, M_{i}\right)}{\sigma\left(u_{n}\right)}\left(\frac{\cos d\left(T u_{n}, x_{i}\right)}{\cos d\left(T u_{0}, x_{i}\right)}+\frac{\cos d\left(T u_{0}, x_{i}\right)}{\cos d\left(T u_{n}, x_{i}\right)}\right)-\frac{1}{2} \sum_{i=1}^{l} \Delta_{i}\left(u_{n}, u_{0}\right) \frac{\cos d\left(T u_{0}, x_{i}\right)}{\cos d\left(T u_{n}, x_{i}\right)} \\
& \geq \sum_{i=1}^{l} \frac{d\left(u_{n}, M_{i}\right)}{\sigma\left(u_{n}\right)} \sqrt{\frac{\cos d\left(T u_{n}, x_{i}\right)}{\cos d\left(T u_{0}, x_{i}\right)} \frac{\cos d\left(T u_{0}, x_{i}\right)}{\cos d\left(T u_{n}, x_{i}\right)}}-\frac{1}{2} \sum_{i=1}^{l} \Delta_{i}\left(u_{n}, u_{0}\right) \frac{\cos d\left(T u_{0}, x_{i}\right)}{\cos d\left(T u_{n}, x_{i}\right)} \\
& =1-\frac{1}{2} \sum_{i=1}^{l} \Delta_{i}\left(u_{n}, u_{0}\right) \frac{\cos d\left(T u_{0}, x_{i}\right)}{\cos d\left(T u_{n}, x_{i}\right)} .
\end{aligned}
$$

Since $\Delta_{i}\left(u_{n}, u_{0}\right) \rightarrow 0$ as $n \rightarrow \infty$ for any $i=1,2, \ldots, l$, we get $\cos d\left(T u_{n}, T u_{0}\right) \rightarrow 1$, which is equivalent that $T u_{n} \rightarrow T u_{0}$. Therefore $T$ is continuous and we complete the proof.

Lemma 3.2. Let $X$ be an admissible complete CAT(1) space having the convex hull finite property. Let $M: X \rightarrow 2^{X}$ be a mapping such that $M(x)$ is a nonempty closed convex subset of $X$ for every $x \in X$ and that

$$
\operatorname{clco} E \subset \bigcup_{x \in E} M(x)
$$

for every finite subset $E$ of $X$. Then, $\left\{M(x) \in 2^{X} \mid x \in X\right\}$ has the finite intersection property. 
Proof. We prove it by contradiction. Suppose that there exists a finite subset

$$
E=\left\{x_{1}, x_{2}, \ldots, x_{l}\right\} \subset X
$$

such that $\bigcap_{i=1}^{l} M\left(x_{i}\right)=\emptyset$. Then, for $u \in \operatorname{cl} \operatorname{co} E$, we can define $g_{u}: \operatorname{cl} \operatorname{co} E \rightarrow[0,+\infty[$ by

$$
g_{u}(y)=-\frac{1}{\sigma(u)} \sum_{i=1}^{l} d\left(u, M\left(x_{i}\right)\right) \log \cos d\left(y, x_{i}\right)
$$

for $y \in X$, where $\sigma(u)=\sum_{i=1}^{l} d\left(u, M\left(x_{i}\right)\right)$. By Lemma 3.1, $g_{u}$ has a unique minimizer $T u \in \operatorname{clco} E$ for every $u \in X$, and the single-valued mapping $T: \operatorname{clco} E \rightarrow \operatorname{clco} E$ is continuous. By the convex hull finite property of $X, T$ has a fixed point $u_{0} \in \operatorname{clco} E$. Since $\bigcap_{i=1}^{l} M\left(x_{i}\right)=\emptyset$, we get $d\left(u_{0}, M\left(x_{i}\right)\right)>0$ for some $i=1,2, \ldots, l$. By permutation, we may assume that $d\left(u_{0}, M\left(x_{i}\right)\right)>0$ for $i=1,2, \ldots, l_{0}$ and $d\left(u_{0}, M\left(x_{i}\right)\right)=0$ for $i=$ $l_{0}+1, l_{0}+2, \ldots, l$. Let $E_{0}=\left\{x_{1}, x_{2}, \ldots, x_{l_{0}}\right\} \subset E$ and $P: \operatorname{clco} E \rightarrow \operatorname{clco} E_{0}$ be the metric projection onto $\mathrm{clco} E_{0}$. Then, from the property of $P$, we have

$$
\cos d\left(u_{0}, v\right) \leq \cos d\left(u_{0}, P u_{0}\right) \cos d\left(P u_{0}, v\right) \leq \cos d\left(P u_{0}, v\right)
$$

for all $v \in \operatorname{cl} \operatorname{co} E_{0}$. In particular, $\cos d\left(u_{0}, x_{i}\right) \leq \cos d\left(P u_{0}, x_{i}\right)$ for $i=1,2, \ldots, l_{0}$. It implies that

$$
\begin{aligned}
g_{u_{0}}\left(P u_{0}\right) & =-\frac{1}{\sigma\left(u_{0}\right)} \sum_{i=1}^{l} d\left(u_{0}, M\left(x_{i}\right)\right) \log \cos d\left(P u_{0}, x_{i}\right) \\
& =-\frac{1}{\sigma\left(u_{0}\right)} \sum_{i=1}^{l_{0}} d\left(u_{0}, M\left(x_{i}\right)\right) \log \cos d\left(P u_{0}, x_{i}\right) \\
& \leq-\frac{1}{\sigma\left(u_{0}\right)} \sum_{i=1}^{l_{0}} d\left(u_{0}, M\left(x_{i}\right)\right) \log \cos d\left(u_{0}, x_{i}\right)=g_{u_{0}}\left(u_{0}\right)
\end{aligned}
$$

and hence $P u_{0}$ minimizes $g_{u_{0}}$ on cl co $E$. From the uniqueness of the minimizer of $g_{u_{0}}$, we obtain $u_{0}=P u_{0}$, and by assumption we have

$$
u_{0}=P u_{0} \in \operatorname{clco} E_{0} \subset \bigcup_{i=1}^{l_{0}} M\left(x_{i}\right) .
$$

On the other hand, since $d\left(u_{0}, M\left(x_{i}\right)\right)>0$ for $i=1,2, \ldots, l_{0}$, we have $u_{0} \notin \bigcup_{i=1}^{l_{0}} M\left(x_{i}\right)$. This is a contradiction and we finish the proof.

Before proving the next result, we remark several facts about $\Delta$-compactness of a subset of admissible complete CAT(1) space $X$. Let $\left\{x_{\alpha}\right\}$ be a net in $X$ with a directed index set $D$. Then, its asymptotic center $A\left(\left\{x_{\alpha}\right\}\right)$ is defined by

$$
A\left(\left\{x_{\alpha}\right\}\right)=\left\{\begin{array}{l|l}
\left.z \in X \mid \limsup _{\alpha \in D} d\left(x_{\alpha}, z\right)=\inf _{y \in X} \limsup _{\alpha \in D} d\left(x_{\alpha}, y\right)\right\} .
\end{array}\right.
$$

If every subnet of $\left\{x_{\alpha}\right\}$ has an identical asymptotic center $\left\{x_{0}\right\}$, then we say $\left\{x_{\alpha}\right\}$ is $\Delta$ convergent to $x_{0} \in X$.

Kirk and Massa [12] showed the following.

Theorem 3.4 (Kirk-Massa [12]). Let $\left\{x_{\alpha}\right\}$ be a universal net in a Banach space $X$. Then, every subnet of $\left\{x_{\alpha}\right\}$ has an identical asymptotic center, that is,

$$
A\left(\left\{x_{\alpha}\right\}\right)=A\left(\left\{x_{\alpha_{\beta}}\right\}\right)
$$

for every subnet $\left\{x_{\alpha_{\beta}}\right\}$ of $\left\{x_{\alpha}\right\}$. 
They proved this result in the setting of Banach spaces, however, the proof is valid also in a general metric space. Further, if $C$ is a subset of a complete CAT(1) space such that $\inf _{y \in C} \sup _{x \in C} d(x, y)<\pi / 2$, then the asymptotic center of a net in $C$ is always a singleton [6]. Since every net in $C$ has a universal subnet, we conclude that $\left\{x_{\alpha}\right\}$ in $C$ has a $\Delta$-convergent subnet.

We also know that if a net $\left\{x_{\alpha}\right\}$ of an admissible CAT(1) space $X$ is $\Delta$-convergent to $x_{0} \in X$, then

$$
x_{0} \in \bigcap_{\alpha \in D} \operatorname{clco}\left\{x_{\beta} \in X \mid \beta \geq \alpha\right\} .
$$

It is implied by a similar method shown in $[6,10]$, which is the case of a sequence.

Lemma 3.3. Let $X$ be an admissible complete CAT(1) space, $C$ a closed convex subset of $X$ such that $\inf _{y \in C} \sup _{x \in C} d(x, y)<\pi / 2$, and $\left\{M_{i} \in 2^{X} \mid i \in I\right\}$ be a family of closed convex subsets of $C$ having the finite intersection property. Then

$$
\bigcap_{i \in I} M_{i} \neq \emptyset \text {. }
$$

Proof. Let $D$ be the family of all finite subsets of the index set $I$ and define a binary relation $\leq$ on $D$ by $\alpha \leq \beta$ if and only if $\alpha \subset \beta$ for all $\alpha, \beta \in D$. Then, $(D, \leq)$ is a directed set. Let $\left\{M_{i_{1}}, M_{i_{2}}, \ldots, M_{i_{n}}\right\}$ be a finite family of $\left\{M_{i}\right\}$ for $\alpha=\left\{i_{1}, i_{2}, \ldots, i_{n}\right\} \in D$. Since $\left\{M_{i}\right\}$ has the finite intersection property, we can choose

$$
x_{\alpha} \in \bigcap_{i \in \alpha} M_{i}=\bigcap_{j=1}^{n} M_{i_{j}} .
$$

Taking such $x_{\alpha}$ for every $\alpha \in D$, we get a net $\left\{x_{\alpha}\right\}$ in $C$ with the index set $D$. Then, by the condition of $C,\left\{x_{\alpha}\right\}$ has a $\Delta$-convergent subnet $\left\{x_{\alpha_{\beta}}\right\}$ with the index directed set $D^{\prime}$. We denote the $\Delta$-limit of $\left\{x_{\alpha_{\beta}}\right\}$ by $x_{0}$ and we show $x_{0} \in \bigcap_{i \in I} M_{i}$. Fix $i \in I$ arbitrarily and let $\alpha=\{i\} \in D$. Then, from the definition of subnet, there exists $\beta_{0} \in D^{\prime}$ such that $\alpha \leq \alpha_{\beta}$ whenever $\beta \in D^{\prime}$ satisfies $\beta_{0} \leq \beta$. Then, we have

$$
x_{\alpha_{\beta}} \in \bigcap_{j \in \alpha_{\beta}} M_{j} \subset \bigcap_{j \in \alpha} M_{j}=M_{i}
$$

for all $\beta \in D^{\prime}$ with $\beta_{0} \leq \beta$. Since $M_{i}$ is closed and convex, taking $\Delta$-limit of the net $\left\{x_{\alpha_{\beta}}\right\}$, we obtain

$$
x_{0}=\Delta-\lim _{\beta \in D^{\prime}} x_{\alpha_{\beta}} \in M_{i} .
$$

Since $i \in I$ is arbitrary, we get $x_{0} \in \bigcap_{i \in I} M_{i}$, which is the desired result.

\section{RESOLVENTS FOR AN EQUILIBRIUM PROBLEM}

In this section, we define a resolvent operator of a bifunction for an equilibrium problem on an admissible complete CAT(1) space.

We begin with the following theorem, which is important for our main result in this section. This result guarantees that the resolvent operator, which will be defined in Theorem 4.6, has the whole space as its effective domain.

Theorem 4.5. Let $X$ be an admissible complete CAT(1) space having the convex hull finite property and $K$ a nonempty closed convex subset of $X$. Suppose that $f: K \times K \rightarrow \mathbb{R}$ satisfies the conditions (E1)-(E4) described above. Then, for any $x \in X$, there exists $z_{0} \in K$ such that

$$
f\left(z_{0}, y\right)-\log \cos d(x, y)+\log \cos d\left(x, z_{0}\right) \geq 0
$$

for every $y \in K$. 
To prove this result, we employ the technique used in the proofs of the corresponding results in $[3,9]$.

Proof. For arbitrarily fixed $x \in X$, let

$$
h(z, y)=f(z, y)-\log \cos d(x, y)+\log \cos d(x, z)
$$

for $z, y \in K$. Notice that $h$ also satisfies the conditions (E1)-(E4). We prove the existence of $z_{0}$ such that $h\left(z_{0}, y\right) \geq 0$ for all $y \in K$.

We will prove it in the following four steps.

Step 1. Determine a subset $C$ of $X$ satisfying the assumption in Lemma 3.3.

Let $u=P_{K} x \in K$, where $P_{K}$ is the metric projection onto $K$. Then we have

$$
\cos d(u, z) \geq \cos d(x, u) \cos d(u, z) \geq \cos d(x, z)
$$

and thus

$$
\log \cos d(u, z) \geq \log \cos d(x, z)
$$

for every $z \in K$. On the other hand, since $f(u, \cdot): K \rightarrow \mathbb{R}$ is a convex function, by Theorem 2.3, it is bounded below. Thus $L=\inf _{y \in K} f(u, y)+\log \cos d(x, u)$ satisfies

$$
-\infty<L \leq f(u, u)+\log \cos d(x, u)=\log \cos d(x, u) \leq 0 .
$$

We may assume $L<0$; if $L=0$, then $z_{0}=u$ satisfies the conclusion. We also have

$$
\begin{aligned}
h(z, u) & =f(z, u)-\log \cos d(x, u)+\log \cos d(x, z) \\
& \leq-f(u, z)-\log \cos d(x, u)+\log \cos d(u, z) \leq-L+\log \cos d(u, z)
\end{aligned}
$$

for all $z \in K$. Thus, if $z \in K$ satisfies $-L+\log \cos d(u, z)=0$, or equivalently, $d(u, z)=$ $\arccos e^{L}$, then $h(z, u) \leq 0$. Let

$$
C=\{z \in K \mid-L+\log \cos d(u, z) \geq 0\}=\left\{z \in K \mid d(u, z) \leq \arccos e^{L}\right\} .
$$

Then $C$ is a closed convex subset of $K$ satisfying

$$
\inf _{v \in C} \sup _{z \in C} d(v, z) \leq \sup _{z \in C} d(u, z) \leq \arccos e^{L}<\frac{\pi}{2} .
$$

Step 2. Define $\{M(y)\}$ and show that their intersection is nonempty.

For $y \in C$, define a subset $M(y)$ of $C$ by

$$
M(y)=\{v \in C \mid h(y, v) \leq 0\} .
$$

Then, since $h(y, \cdot): C \rightarrow \mathbb{R}$ is lower semicontinuous and convex, $M(y)$ is closed and convex. Further, it is nonempty since $y \in M(y)$. To prove that $\{M(y) \mid y \in C\}$ has the finite intersection property, let $E=\left\{y_{1}, y_{2}, \ldots, y_{n}\right\} \subset C$ be an arbitrary finite subset of $C$ and show that cl co $E \subset \bigcup_{i=1}^{n} M\left(y_{i}\right)$. To obtain this inclusion, it is sufficient to show that

$$
\operatorname{co} E \subset \bigcup_{i=1}^{n} M\left(y_{i}\right)
$$

since $\bigcup_{i=1}^{n} M\left(y_{i}\right)$ is closed. We note that the convex hull of $E$ is defined as co $E=\bigcup_{j=1}^{\infty} F_{j}$, where $F_{1}=E$ and $F_{j+1}=\left\{t u_{j} \oplus(1-t) u_{j}^{\prime} \in X \mid u_{j}, u_{j}^{\prime} \in F_{j}, t \in[0,1]\right\}$ for $j \in \mathbb{N}$. We show the following statement by induction: For every $j \in \mathbb{N}$, if $v \in F_{j}$, then there exists $\left\{\mu_{1}, \mu_{2}, \ldots, \mu_{n}\right\} \subset[0,1]$ such that

$$
h\left(y_{k}, v\right) \leq \sum_{i=1}^{n} \mu_{i} h\left(y_{k}, y_{i}\right)
$$

for any $k=1,2, \ldots, n$, and $\sum_{i=1}^{n} \mu_{i}=1$. 
Suppose $j=1$. Then, if $v \in F_{1}=E$, then $v=y_{i_{0}}$ for some $i_{0} \in\{1,2, \ldots, n\}$. Thus letting

$$
\mu_{i}= \begin{cases}1 & \left(i=i_{0}\right) \\ 0 & \left(i \neq i_{0}\right)\end{cases}
$$

we have

$$
h\left(y_{k}, v\right)=h\left(y_{k}, y_{i_{0}}\right)=\sum_{i=1}^{n} \mu_{i} h\left(y_{k}, y_{i}\right)
$$

Next we assume that the statement above holds for fixed $j \in \mathbb{N}$. If $v \in F_{j+1}$, then $v=$ $t u_{j} \oplus(1-t) u_{j}^{\prime}$ for some $u_{j}, u_{j}^{\prime} \in F_{j}$ and $t \in[0,1]$. Then, from the assumption, there exist $\left\{\nu_{1}, \nu_{2}, \ldots, \nu_{n}\right\},\left\{\nu_{1}^{\prime}, \nu_{2}^{\prime}, \ldots, \nu_{n}^{\prime}\right\} \subset[0,1]$ such that

$$
h\left(y_{k}, u_{j}\right) \leq \sum_{i=1}^{n} \nu_{i} h\left(y_{k}, y_{i}\right) \text { and } h\left(y_{k}, u_{j}^{\prime}\right) \leq \sum_{i=1}^{n} \nu_{i}^{\prime} h\left(y_{k}, y_{i}\right)
$$

for any $k=1,2, \ldots, n$, with $\sum_{i=1}^{n} \nu_{i}=\sum_{i=1}^{n} \nu_{i}^{\prime}=1$. By the convexity of $h\left(y_{k}, \cdot\right)$, we have

$$
\begin{aligned}
h\left(y_{k}, v\right) & =h\left(y_{k}, t u_{j} \oplus(1-t) u_{j}^{\prime}\right) \leq t h\left(y_{k}, u_{j}\right)+(1-t) h\left(y_{k}, u_{j}^{\prime}\right) \\
& \leq t \sum_{i=1}^{n} \nu_{i} h\left(y_{k}, y_{i}\right)+(1-t) \sum_{i=1}^{n} \nu_{i}^{\prime} h\left(y_{k}, y_{i}\right)=\sum_{i=1}^{n}\left(t \nu_{i}+(1-t) \nu_{i}^{\prime}\right) h\left(y_{k}, y_{i}\right)
\end{aligned}
$$

for any $k=1,2, \ldots, n$. Therefore, letting $\mu_{i}=t \nu_{i}+(1-t) \nu_{i}^{\prime}$ for $i=1,2, \ldots, n$, we obtain the conclusion. Namely, for every $v \in \operatorname{co} E$, there exists $\left\{\mu_{1}, \mu_{2}, \ldots, \mu_{n}\right\} \subset[0,1]$ such that $h\left(y_{k}, v\right) \leq \sum_{i=1}^{n} \mu_{i} h\left(y_{k}, y_{i}\right)$ for $k=1,2, \ldots, n$, and $\sum_{i=1}^{n} \mu_{i}=1$. Now we show that co $E \subset \bigcup_{i=1}^{n} M\left(y_{i}\right)$ by contradiction. Suppose there exists $v \in \operatorname{co} E$ such that $h\left(y_{k}, v\right)>0$ for every $k=1,2, \ldots, n$. Then, for some $\left\{\mu_{1}, \mu_{2}, \ldots, \mu_{n}\right\} \subset[0,1]$ with $\sum_{i=1}^{n} \mu_{i}=1$, we have

$$
h\left(y_{k}, v\right) \leq \sum_{i=1}^{n} \mu_{i} h\left(y_{k}, y_{i}\right)
$$

for every $k=1,2, \ldots, n$. It implies that

$$
0<\sum_{k=1}^{n} \mu_{k} h\left(y_{k}, v\right) \leq \sum_{k=1}^{n} \sum_{i=1}^{n} \mu_{k} \mu_{i} h\left(y_{k}, y_{i}\right)=\frac{1}{2} \sum_{k=1}^{n} \sum_{i=1}^{n} \mu_{k} \mu_{i}\left(h\left(y_{k}, y_{i}\right)+h\left(y_{i}, y_{k}\right)\right) \leq 0 \text {. }
$$

This is a contradiction and thus we have $h\left(y_{k}, v\right) \leq 0$ for some $k$, that is, $v \in M\left(y_{k}\right)$. Hence we have

$$
\operatorname{clco} E \subset \bigcup_{y \in E} M(y)
$$

for every finite subset $E$ of $C$. It follows from Lemma 3.2 that $\{M(y)\}$ has the finite intersection property, and by Lemma 3.3, we obtain

$$
\bigcap_{y \in C} M(y) \neq \emptyset
$$

Step 3. Prove $h\left(z_{0}, y\right) \geq 0$ for every $y \in C$.

Since $\bigcap_{y \in C} M(y)$ is nonempty, we can take $z_{0} \in \bigcap_{y \in C} M(y)$. Then we obtain $h\left(y, z_{0}\right) \leq$ 0 for every $y \in C$. For arbitrarily fixed $w \in C$, let $y_{t}=t w \oplus(1-t) z_{0} \in C$ with $\left.t \in\right] 0,1[$. Then, we have

$$
0=h\left(y_{t}, y_{t}\right)=h\left(y_{t}, t w \oplus(1-t) z_{0}\right) \leq t h\left(y_{t}, w\right)+(1-t) h\left(y_{t}, z_{0}\right) \leq t h\left(y_{t}, w\right),
$$

which implies $h\left(y_{t}, w\right) \geq 0$. Then, since $h(\cdot, w)$ is upper hemicontinuous, letting $t \rightarrow 0$, we have

$$
h\left(z_{0}, w\right) \geq \limsup _{t \rightarrow 0} h\left(y_{t}, w\right) \geq 0 .
$$

Since $w \in C$ is arbitrary, we obtain $h\left(z_{0}, y\right) \geq 0$ for every $y \in C$. 
Step 4 . Prove $h\left(z_{0}, y\right) \geq 0$ for every $y \in K$.

Since $u=P_{K} x$ as described in Step 1, by the definition of $C$ with the fact that $z_{0} \in C$, we have $d\left(u, z_{0}\right) \leq \arccos e^{L}$. Let

$$
u_{0}= \begin{cases}u & \left(d\left(u, z_{0}\right)=\arccos e^{L}\right) \\ z_{0} & \left(d\left(u, z_{0}\right)<\arccos e^{L}\right) .\end{cases}
$$

Then, since $h(z, u) \leq 0$ whenever $d(u, z)=\arccos e^{L}$ as in Step 1 , and $h\left(z_{0}, z_{0}\right)=0$, we have

$$
h\left(z_{0}, u_{0}\right) \leq 0 .
$$

On the other hand, by the definition of $u_{0}$, we also have $d\left(u_{0}, u\right)<\arccos e^{L}$, or equivalently, $\cos d\left(u_{0}, u\right)>e^{L}$.

Fix $y \in K \backslash C$ arbitrarily. Then, by taking $\left.t_{0} \in\right] 0,1[$ sufficiently small, we can obtain

$$
\cos d\left(t_{0} y \oplus\left(1-t_{0}\right) u_{0}, u\right) \geq t_{0} \cos d(y, u)+\left(1-t_{0}\right) \cos d\left(u_{0}, u\right)>e^{L},
$$

that is, $d\left(t_{0} y \oplus\left(1-t_{0}\right) u_{0}, u\right)<\arccos e^{L}$ and hence $t_{0} y \oplus\left(1-t_{0}\right) u_{0} \in C$. By the convexity of $h\left(z_{0}, \cdot\right)$, it implies that

$$
0 \leq h\left(z_{0}, t_{0} y \oplus\left(1-t_{0}\right) u_{0}\right) \leq t_{0} h\left(z_{0}, y\right)+\left(1-t_{0}\right) h\left(z_{0}, u_{0}\right)
$$

and hence

$$
h\left(z_{0}, y\right) \geq-\frac{1-t_{0}}{t_{0}} h\left(z_{0}, u_{0}\right) \geq 0 .
$$

Since $y \in K \backslash C$ is arbitrary, we conclude that $h\left(z_{0}, y\right) \geq 0$ for every $y \in K$.

We now define a resolvent operator $R_{f}$ for a bifunction $f: K \times K \rightarrow \mathbb{R}$ satisfying the conditions (E1)-(E4). As in the following theorem, it is defined as a single-valued mapping and its domain is the whole space $X$.

Theorem 4.6. Let $X$ be an admissible complete CAT(1) space having the convex hull finite property, $K$ a nonempty closed convex subset of $X$. Suppose that $f: K \times K \rightarrow \mathbb{R}$ satisfies the conditions (E1)-(E4). For each $x \in X$, define a subset $R_{f} x$ of $K$ by

$$
R_{f} x=\left\{z \in K \mid \inf _{y \in K}(f(z, y)-\log \cos d(x, y)+\log \cos d(x, z)) \geq 0\right\} .
$$

Then, the following hold:

(i) $R_{f} x$ consists of one point for every $x \in X$, and therefore $R_{f}: X \rightarrow K$ is defined as a single-valued mapping;

(ii) $R_{f}$ satisfies the following inequality for any $x_{1}, x_{2} \in X$ :

$$
\frac{\cos d\left(x_{1}, R_{f} x_{2}\right)}{\cos d\left(x_{1}, R_{f} x_{1}\right)}+\frac{\cos d\left(x_{2}, R_{f} x_{1}\right)}{\cos d\left(x_{2}, R_{f} x_{2}\right)} \leq 2 \cos d\left(R_{f} x_{1}, R_{f} x_{2}\right),
$$

and thus $R_{f}$ is spherically nonspreading of sum type.

(iii) Fix $R_{f}=$ Equil $f$ and it is closed and convex.

Proof. Let $x \in X$. Then, by Theorem 4.5 , there exists $z_{0} \in K$ such that

$$
f\left(z_{0}, y\right)-\log \cos d(x, y)+\log \cos d\left(x, z_{0}\right) \geq 0
$$

for all $y \in K$ and thus $z_{0} \in R_{f} x$. This implies that $R_{f} x$ is nonempty.

Let $z \in R_{f} x$ and fix $w \in K$ arbitrarily. If $w \neq z$, then since $t w \oplus(1-t) z \in K$ for $\left.t \in\right] 0,1$, we have

$$
\begin{aligned}
0 \leq & f(z, t w \oplus(1-t) z)-\log \cos d(x, t w \oplus(1-t) z)+\log \cos d(x, z) \\
\leq & t f(z, w)+(1-t) f(z, z) \\
& -\log (\cos d(x, w) \sin (t d(w, z))+\cos d(x, z) \sin ((1-t) d(w, z)))
\end{aligned}
$$




$$
\begin{aligned}
& +\log \sin d(w, z)+\log \cos d(x, z) \\
= & t f(z, w)+L(t)
\end{aligned}
$$

where

$$
\begin{aligned}
L(t)= & -\log (\cos d(x, w) \sin (t d(w, z))+\cos d(x, z) \sin ((1-t) d(w, z))) \\
& +\log (\cos d(x, z) \sin d(w, z)) .
\end{aligned}
$$

Notice that $L(t) \rightarrow 0$ as $t \downarrow 0$. By l'Hospital's rule, we have

$$
0 \leq f(z, w)+\lim _{t \downarrow 0} \frac{L(t)}{t}=f(z, w)+\lim _{t \downarrow 0} \frac{d L(t)}{d t} .
$$

Since

$$
\begin{aligned}
\frac{d L(t)}{d t} & =\frac{d(w, z)(-\cos d(x, w) \cos (t d(w, z))+\cos d(x, z) \cos ((1-t) d(w, z)))}{\cos d(x, w) \sin (t d(w, z))+\cos d(x, z) \sin ((1-t) d(w, z))} \\
& \rightarrow \frac{d(w, z)((-\cos d(x, w)+\cos d(x, z) \cos d(w, z))}{\cos d(x, z) \sin d(w, z)} \\
& =\frac{d(w, z)}{\sin d(w, z)}\left(\cos d(w, z)-\frac{\cos d(x, w)}{\cos d(x, z)}\right)
\end{aligned}
$$

as $t \downarrow 0$, we have

$$
0 \leq f(z, w)+\frac{d(w, z)}{\sin d(w, z)}\left(\cos d(w, z)-\frac{\cos d(x, w)}{\cos d(x, z)}\right)
$$

for $x \in X, z \in R_{f} x$, and $w \in K$ such that $w \neq z$.

To prove (i) and (ii), let $x_{1}, x_{2} \in X, z_{1} \in R_{f} x_{1}, z_{2} \in R_{f} x_{2}$, We first suppose that $z_{1} \neq z_{2}$. Then, since $z_{1}, z_{2} \in K$, it follows from the previous inequality that

$$
\begin{aligned}
0 & \leq f\left(z_{1}, z_{2}\right)+\frac{d\left(z_{2}, z_{1}\right)}{\sin d\left(z_{2}, z_{1}\right)}\left(\cos d\left(z_{2}, z_{1}\right)-\frac{\cos d\left(x_{1}, z_{2}\right)}{\cos d\left(x_{1}, z_{1}\right)}\right), \\
0 & \leq f\left(z_{2}, z_{1}\right)+\frac{d\left(z_{1}, z_{2}\right)}{\sin d\left(z_{1}, z_{2}\right)}\left(\cos d\left(z_{1}, z_{2}\right)-\frac{\cos d\left(x_{2}, z_{1}\right)}{\cos d\left(x_{2}, z_{2}\right)}\right) .
\end{aligned}
$$

Adding their both sides, we have

$$
\begin{aligned}
0 & \leq f\left(z_{1}, z_{2}\right)+f\left(z_{2}, z_{1}\right)+\frac{d\left(z_{1}, z_{2}\right)}{\sin d\left(z_{1}, z_{2}\right)}\left(2 \cos d\left(z_{1}, z_{2}\right)-\frac{\cos d\left(x_{2}, z_{1}\right)}{\cos d\left(x_{2}, z_{2}\right)}-\frac{\cos d\left(x_{1}, z_{2}\right)}{\cos d\left(x_{1}, z_{1}\right)}\right) \\
& \leq \frac{d\left(z_{1}, z_{2}\right)}{\sin d\left(z_{1}, z_{2}\right)}\left(2 \cos d\left(z_{1}, z_{2}\right)-\frac{\cos d\left(x_{2}, z_{1}\right)}{\cos d\left(x_{2}, z_{2}\right)}-\frac{\cos d\left(x_{1}, z_{2}\right)}{\cos d\left(x_{1}, z_{1}\right)}\right) .
\end{aligned}
$$

Since $d\left(z_{1}, z_{2}\right) / \sin d\left(z_{1}, z_{2}\right)>0$, we have

$$
\frac{\cos d\left(x_{2}, z_{1}\right)}{\cos d\left(x_{2}, z_{2}\right)}+\frac{\cos d\left(x_{1}, z_{2}\right)}{\cos d\left(x_{1}, z_{1}\right)} \leq 2 \cos d\left(z_{1}, z_{2}\right) .
$$

Note that this inequality is obviously true if $z_{1}=z_{2}$. Using this inequality, we may show that $R_{f} x$ is a singleton; if $x=x_{1}=x_{2}$, then

$$
2 \cos d\left(z_{1}, z_{2}\right) \geq \frac{\cos d\left(x, z_{1}\right)}{\cos d\left(x, z_{2}\right)}+\frac{\cos d\left(x, z_{2}\right)}{\cos d\left(x, z_{1}\right)} \geq 2 \sqrt{\frac{\cos d\left(x, z_{1}\right)}{\cos d\left(x, z_{2}\right)} \frac{\cos d\left(x, z_{2}\right)}{\cos d\left(x, z_{1}\right)}}=2,
$$

and thus $\cos d\left(z_{1}, z_{2}\right)=1$, which implies that $z_{1}=z_{2}$. Hence $R_{f} x$ is always a singleton and (i) holds. From this fact, we can write $z_{1}=R_{f} x_{1}$ and $z_{2}=R_{f} x_{2}$ in (4.5). Thus we obtain the inequality in (ii). In addition, we have

$$
\begin{aligned}
2 \cos d\left(R_{f} x_{1}, R_{f} x_{2}\right) & \geq \frac{\cos d\left(x_{1}, R_{f} x_{2}\right)}{\cos d\left(x_{1}, R_{f} x_{1}\right)}+\frac{\cos d\left(x_{2}, R_{f} x_{1}\right)}{\cos d\left(x_{2}, R_{f} x_{2}\right)} \\
& \geq \cos d\left(x_{1}, R_{f} x_{2}\right)+\cos d\left(x_{2}, R_{f} x_{1}\right),
\end{aligned}
$$


which shows that $R_{f}$ is spherically nonspreading of sum type.

For (iii), suppose $z \in \operatorname{Fix} R_{f}$, or equivalently, $z=R_{f} z$. Using the inequality (4.4), we have

$$
0 \leq f(z, w)+\frac{d(w, z)}{\sin d(w, z)}\left(\cos d(w, z)-\frac{\cos d(z, w)}{\cos d(z, z)}\right)=f(z, w)
$$

for every $w \in K$ with $w \neq z$. Since $f(z, w)=0$ if $z=w$, we get $f(z, w) \geq 0$ for all $w \in K$. It implies that $z \in$ Equil $f$. On the other hand, if $z \in$ Equil $f$, then, since $-\log \cos t \geq 0$ for any $t \in[0, \pi / 2[$, we have

$$
\begin{aligned}
\inf _{y \in K}(f(z, y)-\log \cos d(z, y)+\log \cos d(z, z)) & =\inf _{y \in K}(f(z, y)-\log \cos d(z, y)) \\
& \geq \inf _{y \in K} f(z, y) \geq 0 .
\end{aligned}
$$

This inequality shows that $z=R_{f} z$, that is $z \in \operatorname{Fix} R_{f}$. Hence we have $\operatorname{Fix} R_{f}=$ Equil $f$. Further, since a spherically nonspreading mapping $R_{f}$ of sum type is quasinonexpansive if it has a fixed point, we conclude that Fix $R_{f}$ is closed and convex.

\section{EXISTENCE AND APPROXIMATING METHODS OF THE SOLUTIONS}

As mentioned in the previous section, a resolvent operator of a bifunction $f$ with the conditions (E1)-(E4) is spherically nonspreading of sum type, and the set of solutions to the equilibrium problem for $f$ coincides with the set of fixed points of its resolvent. Therefore, we may apply fixed point theorems and approximation theorems for spherically nonspreading mappings to the existence and the approximation of the solutions to the equilibrium problem.

Notice that every spherically nonspreading mapping of sum type is also that of product type; see Section 2.

In a similar way to $\Delta$-convergence of a net, we say that a sequence $\left\{x_{n}\right\}$ of an admissible CAT(1) space $X$ is $\Delta$-convergent to $x_{0} \in X$ if $\left\{x_{0}\right\}=A\left(\left\{x_{n_{k}}\right\}\right)$, or equivalently,

$$
\limsup _{k \rightarrow \infty} d\left(x_{n_{k}}, x_{0}\right)=\inf _{y \in X} \limsup _{k \rightarrow \infty} d\left(x_{n_{k}}, y\right)
$$

for every subsequence $\left\{x_{n_{k}}\right\}$ of $\left\{x_{n}\right\}$.

Theorem 5.7 (Kimura-Kohsaka [10]). Let $X$ be an admissible complete CAT(1) space such that $\operatorname{diam} X<\pi / 2$. Let $T: X \rightarrow X$ be a spherically nonspreading mapping of product type. Then Fix $T \neq \emptyset$ and a sequence $\left\{T^{n} x\right\}$ is $\Delta$-convergent to some $x_{0} \in$ Fix $T$ for each $x \in X$.

From this result, we can deduce the existence and approximation theorem of the solutions to an equilibrium problem as follows:

Theorem 5.8. Let $X$ be an admissible complete CAT(1) space and suppose that it has the convex hull finite property and $\operatorname{diam} X<\pi / 2$. Let $K$ be a nonempty closed convex subset of $X$. Suppose that $f: K \times K \rightarrow \mathbb{R}$ satisfies the conditions (E1)-(E4) described above. Then Equil $f \neq \emptyset$ and the sequence $\left\{R_{f}^{n} x\right\}$ is $\Delta$-convergent to some $x_{0} \in$ Equil $f$ for each $x \in X$.

Proof. The resolvent $R_{f}$ of a bifunction $f$ is a selfmapping on $X$. Since it is a spherically nonspreading mapping of sum type, it is also that of product type. Thus, by Theorem 5.7, it has a fixed point and $\left\{R_{f}^{n} x\right\}$ is $\Delta$-convergent to some $x_{0} \in$ Fix $R_{f}$. Consequently, by Theorem 4.6 (iii), we have Equil $f=$ Fix $R_{f} \neq \emptyset$ and thus we obtain the desired result.

At the end of this section, we mention equilibrium problems on a subset of the unit sphere $S_{H}$ of a real Hilbert space $H$. Let $X$ be a nonempty closed convex subset of $S_{H}$ such that $d(x, y)<\pi / 2$ for every $x, y \in X$, where the metric $d$ on $X$ is the spherical metric defined on $S_{H}$. In this case, $X$ is an admissible complete CAT(1) space as well as it has the convex hull finite property. We can show this fact by the following. 
Theorem 5.9 (Ariza-Ruiz-Li-Acedo [2]). Let $X$ be a CAT(1) space and $K$ a nonempty closed convex subset of $X$ such that $\operatorname{diam} K<\pi / 2$. Suppose that a continuous mapping $T: K \rightarrow K$ satisfies that $\operatorname{cl} T(K)$ is compact. Then, $T$ has a fixed point.

Let $E$ be a finite subset of $X$ and $T: \operatorname{clco} E \rightarrow \operatorname{clco} E$ a continuous mapping. Then, cl co $E$ is included in a finite-dimensional subspace $M$ of $H$ linearly spanned by $E$. It follows that cl co $E$ is compact in $M$ since cl co $E$ is bounded. Thus we have cl co $E$ is also compact with respect to the spherical metric on $X$. Consequently, $T$ has a fixed point by applying Theorem 5.9 with $K=\operatorname{cl} \operatorname{co} E$, and hence $X$ has the convex hull finite property. This fact implies that Theorem 5.8 is valid for such a space.

Acknowledgement. The author is grateful to anonymous referees for the comments and suggestions on the manuscript.

\title{
REFERENCES
}

[1] Alghamdi, M. A.; Kirk W. A.; Shahzad, N., Remarks on convex combinations in geodesic spaces. J. Nonlinear Convex Anal. 15 (2014), 49-59.

[2] Ariza-Ruiz, D.; Li, C.; López-Acedo, G. The Schauder fixed point theorem in geodesic spaces. J. Math. Anal. Appl. 417 (2014), 345-360.

[3] Blum, E.; Oettli, W. From optimization and variational inequalities to equilibrium problems. Math. Student 63 (1994), 123-145.

[4] Bridson, M. R.; Haefliger, A. Metric spaces of non-positive curvature. Grundlehren der Mathematischen Wissenschaften [Fundamental Principles of Mathematical Sciences] 319. Springer-Verlag, Berlin, 1999.

[5] Combettes, P. L.; Hirstoaga, S. A. Equilibrium programming in Hilbert spaces. J. Nonlinear Convex Anal. 6 (2005), 117-136.

[6] Espínola, R.; Fernández-León, A. CAT(k)-spaces, weak convergence and fixed points. J. Math. Anal. Appl. 353 (2009), 410-427.

[7] Kajimura, T.; Kimura, Y. A new definition of resolvents for convex functions on complete geodesic spaces. Study on nonlinear analysis and convex analysis. RIMS Kôkyûroku 2112, Kyoto University, Kyoto, 2019, 141-147.

[8] Khamsi, M. A. KKM and Ky Fan theorems in hyperconvex metric spaces. J. Math. Anal. Appl. 204 (1996), 298-306.

[9] Kimura, Y.; Kishi, Y. Equilibrium problems and their resolvents in Hadamard spaces. J. Nonlinear Convex Anal. 19 (2018), 1503-1513.

[10] Kimura, Y.; Kohsaka, F. Spherical nonspreadingness of resolvents of convex functions in geodesic spaces. J. Fixed Point Theory Appl. 18 (2016), 93-115.

[11] Kimura, Y.; Satô, K. Convergence of subsets of a complete geodesic space with curvature bounded above. Nonlinear Anal. 75 (2012), 5079-5085.

[12] Kirk, W. A.; Massa, S. Remarks on asymptotic and Chebyshev centers. Houston J. Math. 16 (1990), 357-364.

[13] Kumam, P.; Chaipunya, P., Equilibrium problems and proximal algorithms in Hadamard spaces. 2018, arXiv:1807.10900 [math.OC].

[14] Niculescu, C. P.; Rovenţa, I. Fan's inequality in geodesic spaces. Appl. Math. Lett. 22 (2009), 1529-1533.

[15] Shabanian, S.; Vaezpour, S. M. A minimax inequality and its applications to fixed point theorems in CAT(0) spaces. Fixed Point Theory Appl. 2011 2011:61, 9pp.

\author{
TOHO UNIVERSITY \\ DEPARTMENT OF INFORMATION SCIENCE \\ MIYAMA, FUNABASHI, CHIBA 274-8510, JAPAN \\ Email address: yasunorieis.sci.toho-u.ac.jp
}

